Monatsschr Kinderheilkd 2008 • 156:433

DOI 10.1007/s00112-008-1746-3

Online publiziert: 23. April 2008

๑) Springer Medizin Verlag 2008

\section{Reinhardt}

Kinderklinik und Poliklinik, Dr. von Haunersches Kinderspital, Klinikum, Ludwig-Maximilians-Universität München

\title{
Neue Approbationsordnung: Herausforderungen für die Pädiatrie
}

Deutsche Universitäten stehen seit dem von Humboldt geprägten Bildungsideal der Einheit von Forschung und Lehre vor dem Dilemma, einerseits den primär wertfreien Erkenntnisgewinn durch die Forschung vermitteln, andererseits eine berufsbezogene Ausbildung ermöglichen zu wollen. Hinzu kommt in der Medizin noch die strukturgegebene Notwendigkeit, medizinische Versorgung auf höchstem qualitativem Niveau zu gewährleisten. Dieser Konflikt wird weiter dadurch verschärft, dass die Exzellenzinitiative von Bund und Ländern einseitig die Forschung berücksichtigt, während bei der Umsetzung der neuen Approbationsordnung, der leistungsorientierten Mittelvergabe (LOM) und beim zielgerichteten Einsatz der Studiengebühren die Lehrleistung als Wertekriterium einer Fakultät berücksichtigt werden muss.

\section{(2) Bei der Bewertung einer Fakultät muss die Lehrleistung als Kriterium berücksichtigt werden}

Die neue Approbationsordnung hat 2002 an allen medizinischen Fakultäten eine grundlegende Neukonzeption der medizinischen Curricula erforderlich gemacht.

Als Dekan einer großen medizinischen Fakultät konnte ich in den letzten 3 Jahren „,begleitend“ miterleben, dass über eine professionelle Umsetzung wesentliche Ziele dieser neuen Approbationsordnung trotz anfänglicher Skepsis in einem stets „lernenden" System umgesetzt wurden. In der medizinischen Fakultät der Ludwig-Maximilians-Universität München sind dies (Muntau):
- Ausbau des praxisorientierten Unterrichts mit Schwerpunktbildung im Bereich klinischer Fertigkeiten und Kommunikationsfähigkeit, u. a. unter Einsatz von Schauspielerpatienten. Inzwischen macht der Kleingruppenunterricht $70 \%$ aller Lehrveranstaltungen aus. Die Lehrleistung hat sich seit Einführung des neuen Medizinischen Curriculums München (MeCuM) in einigen Modulen vervierfacht.

- Der fächer- und fakultätsübergreifende Einsatz der E-Learning-Plattform CASUS ${ }^{\circledR}$, die in Tutorien, Pathophysiologieveranstaltungen und zur Prüfungsvorbereitung eingesetzt wird.

- Eine innovative Prüfungskonzeption mit neuen Prüfungsformaten wie aufwendigen klinischen praktischen Prüfungen im OSCE-Format („objective structured clinical examination") mit standardisierten Patienten und einer Datenbank evaluierter MultipleChoice(MC)-Prüfungsfragen.

- Eine einheitliche flächendeckende Online-Evaluation von Lehrveranstaltungen mit standardisierten Fragebögen, integriertem Berichtswesen, leistungsorientierter Mittelvergabe auf Fakultätsebene und Vergabe von Lehrpreisen.

- Die Durchführung von hochschuldidaktischen Qualifikationsmaßnahmen für Dozenten. Inzwischen haben über 1000 Fakultätsmitglieder an einem mehrtägigen strukturierten Hochschullehrertraining oder an einem Workshop zur Verbesserung der Prüfungsqualität teilgenommen. Seit 2007 sind entsprechend der inneren Auslegungsbestimmungen diese Kurse Pflicht für die Habilitation.
Bekannterweise führen viele Wege nach Rom. Das Freiburger (Forster), das Heidelberger (Bosse) und das Berliner Modell (Gaedicke) zeigen weitere Lösungsmöglichkeiten auf. Das Ziel, durch Überdenken etablierter Unterrichtsformen die Studierenden nach ihrer individuellen Leistungsfähigkeit zu behandeln und jedem Einzelnen die ihm angemessene Chance zu geben, seine persönlichen Fähigkeiten optimal zu entwickeln, haben jedoch alle 4 Ausbildungssysteme gemeinsam.

Hoffen wir, dass nicht nur die Studierenden und späteren Ärzte, sondern auch die Patienten davon profitieren!

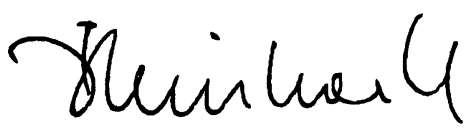

D. Reinhardt

\section{Korrespondenzadresse \\ Prof. Dr. D. Reinhardt \\ Kinderklinik und Poliklinik, \\ Dr. von Haunersches Kinderspital, Klinikum, Ludwig-Maximilians-Universität München Lindwurmstraße 4, 80337 München dietrich.reinhardt@med.uni-muenchen.de}

